\title{
Implementing the AMI READMITS Risk Assessment Score to Increase Referrals Among Patients With Type I Myocardial Infarction
}

Nailya Muganlinskaya, DNP, MPH, ACNP-BC, MSN, Laurie Posey, EdD, Diane Vail Skojec, DNP, ANP-BC, MSN, and Jon Rodney Resar, MD

\section{ABSTRACT}

Objective: Assessing the risk characteristics of patients with acute myocardial infarction (MI) can help providers make appropriate referral decisions. This quality improvement project sought to improve timely, appropriate referrals among patients with type I MI by adding a risk assessment, the AMI READMITS score, to the existing referral protocol.

Methods: Patients' chart data were analyzed to assess changes in referrals and timely follow-up appointments from pre-intervention to intervention. A survey assessed providers' satisfaction with the new referral protocol.

Results: Among 57 patients $(\mathrm{n}=29$ preintervention; $\mathrm{n}$ $=28$ intervention), documented referrals increased significantly from $66 \%$ to $89 \%(\chi 2=4.571, d f=1$, $P=0.033)$; and timely appointments increased by $10 \%$, which was not significant $(\chi 2=3.550, d f=2$, $P=0.169$ ). Most providers agreed that the new protocol was easy to use, useful in making referral decisions, and improved the referral process. All agreed the risk score should be incorporated into electronic clinical notes. Provider opinions related to implementing the risk score in clinical practice were mixed. Qualitative feedback suggests this was due to limited validation of the AMI READMITS score in reducing readmissions.

Conclusions: Our risk-based referral protocol helped to increase appropriate referrals among patients with type I MI. Provider adoption may be enhanced by incorporating the protocol into electronic clinical notes. Research to further validate the accuracy of the AMI READMITS score in predicting readmissions may support adoption of the protocol in clinical practice.

Keywords: quality improvement; type I myocardial infarction; referral process; readmission risk; risk assessment; chart review.
E arly follow-up after discharge is an important strategy to reduce the risk of unplanned hospital readmissions among patients with various conditions..$^{1-3}$ While patient confounding factors, such as chronic health problems, environment, socioeconomic status, and literacy, make it difficult to avoid all unplanned readmissions, early follow-up may help providers identify and appropriately manage some health-related issues, and as such is a pivotal element of a readmission prevention strategy. ${ }^{4}$ There is evidence that patients with non-ST elevation myocardial infarction (NSTEMI) who have an outpatient appointment with a physician within 7 days after discharge have a lower risk of 30-day readmission. ${ }^{5}$

Our hospital's postmyocardial infarction clinic was created to prevent unplanned readmissions within 30 days after discharge among patients with type I myocardial infarction (MI). Since inception, the number of referrals has been much lower than expected. In 2018, the total number of patients discharged from the hospital with type I Ml and any troponin I level above $0.40 \mathrm{ng} / \mathrm{mL}$ was 313. Most of these patients were discharged from the hospital's cardiac units; however, only 91 referrals were made. To increase referrals, the cardiology nurse practitioners (NPs) developed a post-Ml referral protocol (Figure 1). However, this protocol was not consistently used and referrals to the clinic remained low.

Evidence-based risk assessment tools have the potential to increase effective patient management. For

From The Johns Hopkins Hospital, Baltimore, MD (Dr. Muganlinskaya and Dr. Skojec, retired); The George Washington University, Washington, DC (Dr. Posey); and Johns Hopkins University, Baltimore, MD (Dr. Resar). 


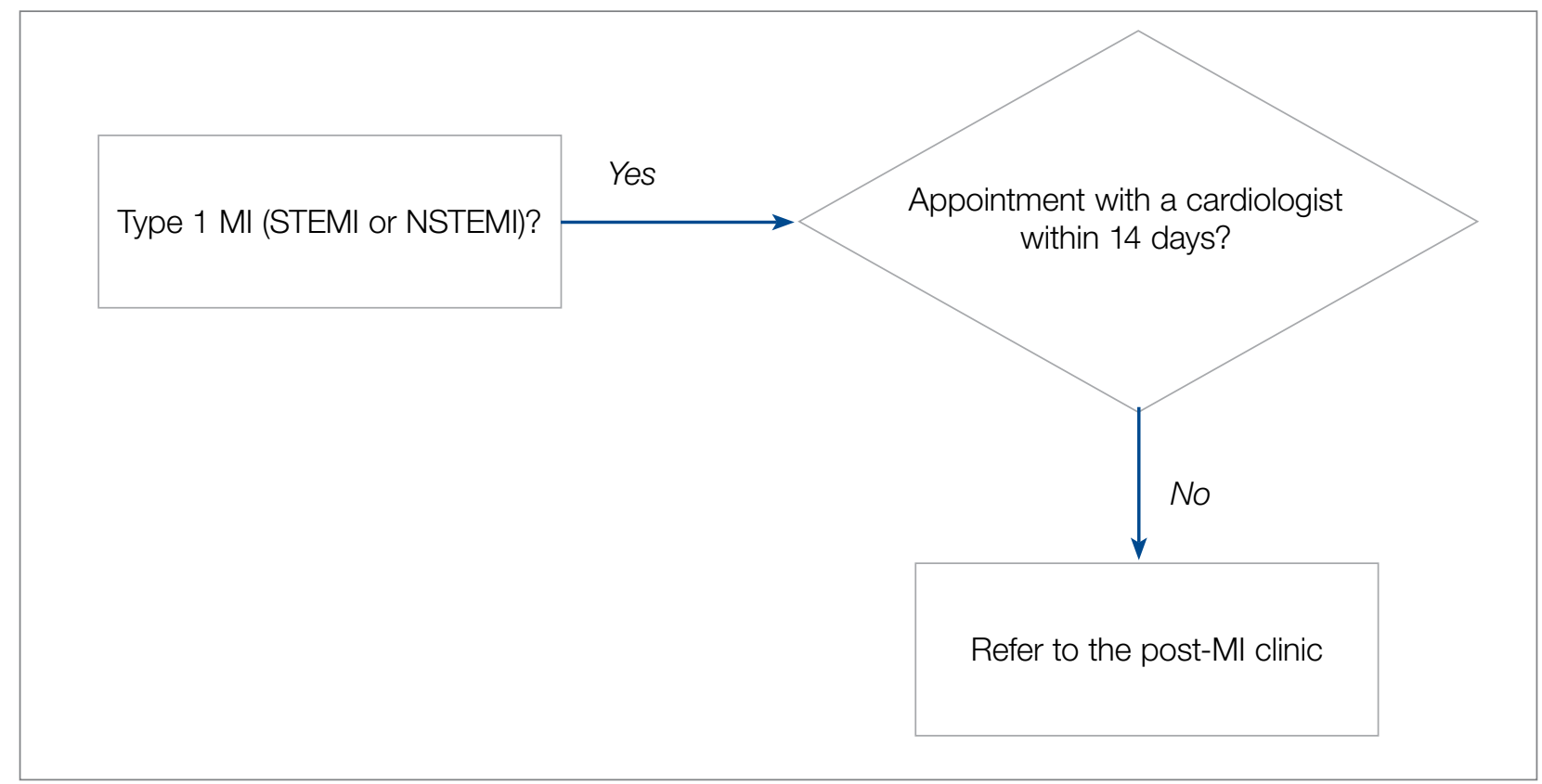

Figure 1. Current referral protocol used to guide the hospital's clinicians to make a referral decision prior to discharge. Clinicians are asked to refer patients to the post-MI clinic if an appointment with a cardiologist is not available within 14 days after discharge. MI, myocardial infarction; NSTEMI, non-ST-segment elevation myocardial infarction; STEMI, ST-segment elevation myocardial infarction.

example, cardiology providers at the hospital utilize various scores, such as $\mathrm{CHA}_{2} \mathrm{DS}_{2}-\mathrm{VASc}^{6}$ and the Society of Thoracic Surgery risk score, ${ }^{7}$ to plan patient management. Among the scores used to predict unplanned readmissions for $\mathrm{Ml}$ patients, the most promising is the AMI READMITS score. ${ }^{8}$ Unlike other nonspecific prediction models, the AMI READMITS score was developed based on variables extracted from the electronic health records (EHRs) of patients who were hospitalized for $\mathrm{Ml}$ and readmitted within 30 days after discharge. Recognizing the potential to increase referrals by integrating an Ml-specific risk assessment, this quality improvement study modified the existing referral protocol to include the patients' AMI READMITS score and recommendations for follow-up.

Currently, there are no clear recommendations on how soon after discharge patients with Ml should undergo follow-up. As research data vary, we selected 7 days follow-up for patients from high risk groups based on the "See you in 7" initiative for patients with heart failure (HF) and Ml, ${ }^{9,10}$ as well as evidence that patients with NSTEMI have a lower risk of 30-day readmission if they have follow-up within 7 days after discharge ${ }^{5}$; and we selected 14 days follow-up for patients from low-risk groups based on evidence that postdischarge follow-up within 14 days reduces risk of 30-day readmission in patients with acute myocardial infarction (AMI) and/or acutely decompensated HF.11

\section{Methods}

This project was designed to answer the following question: For adult patients with type I MI, does implementation of a readmission risk assessment referral protocol increase the percentage of referrals and appointments scheduled within a recommended time? Anticipated outcomes included: (1) increased referrals to a cardiologist or the post-MI clinic; (2) increased scheduled follow-up appointments within 7 to 14 days; (3) provider satisfaction with the usability and usefulness of the new protocol; and (4) consistent provider adoption of the new risk assessment referral protocol.

To evaluate the degree to which these outcomes were achieved, we reviewed patient charts for 2 


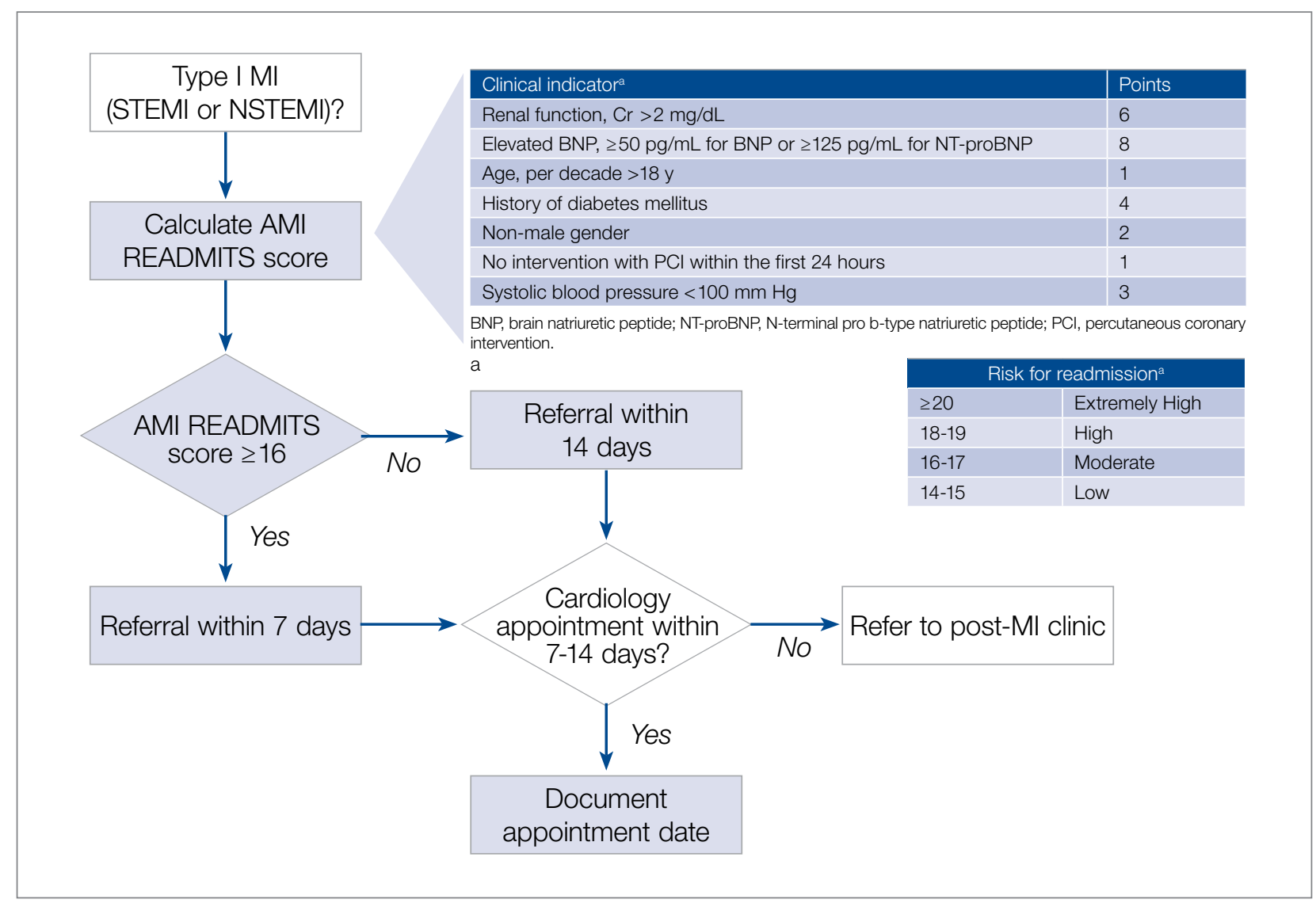

Figure 2. Post-myocardial infarction referral protocol to guide postdischarge referrals process implemented during the study. Shaded boxes represent new process steps. Clinicians were asked to make referrals based on patients' risk for readmission after type I MI based on a calculated risk score. Patients with the risk score $\geq 16$ had to see a cardiologist within 7 days after discharge; patients with the risk score $<16$ had to see a cardiologist within 14 days after discharge. If an appointment with a cardiologist was not available within 7 to 14 days, clinicians were asked to refer patients to the post-Ml clinic.

MI, myocardial infarction; NSTEMI, non-ST-segment elevation myocardial infarction; STEMI, ST-segment elevation myocardial infarction.

${ }^{a}$ Clinical indicators and risk for readmission: Nguyen OK, Makam AN, Clark C, et al. Predicting 30-day hospital readmissions in acute myocardial infarction: the AMI "READMITS" (renal function, elevated brain natriuretic peptide, age, diabetes mellitus, nonmale sex, intervention with timely percutaneous coronary intervention, and low systolic blood pressure) score. J Am Heart Assoc. 2018;7(8):e008882. 2018. doi:10.1161/JAHA.118.008882.

months prior and 2 months during implementation of the new referral protocol. As shown in Figure 2, the new protocol added the following process steps to the existing protocol: calculation of the AMI READMITS score, recommendations for follow-up based on patients' risk score, and guidance to refer patients to the post-Ml clinic if patients did not have an appointment with a cardiologist within 7 to 14 days after discharge. Patients' risk assessment scores were obtained from forms completed by clinicians during the intervention. Clinician's perceptions related to the usability and usefulness of the new protocol and feed- back related to its long-term adoption were assessed using a descriptive survey.

The institutional review board classified this project as a quality improvement project. To avoid potential loss of patient privacy, no identifiable data were collected, a unique identifier unrelated to patients' records was generated for each patient, and data were saved on a password-protected cardiology office computer.

\section{Population}

The project population included all adult patients $(\geq 18$ years old) with type I MI who were admitted or trans- 
ferred to the hospital, had a percutaneous coronary intervention $(\mathrm{PCl})$, or were managed without $\mathrm{PCl}$ and discharged from the hospital's cardiac care unit (CCU) and progressive cardiac care unit (PCCU). The criteria for type I MI included the "detection of a rise and/or fall of cardiac troponin with at least 1 value above the 99th percentile and with at least 1 of the following: symptoms of acute myocardial ischemia; new ischemic electrocardiographic (ECG) changes; development of new pathological Q waves; imaging evidence of new loss of viable myocardium or new regional wall motion abnormality in a pattern consistent with an ischemic etiology; identification of a coronary thrombus by angiography including intracoronary imaging or by autopsy."12 The study excluded patients with type I MI who were referred for coronary bypass surgery.

\section{Intervention}

The revised risk assessment protocol was implemented within the CCU and PCCU. The lead investigator met with each provider to discuss the role of the post-MI clinic, current referral rates, the purpose of the project, and the new referral process to be completed during the project for each patient discharged with type I MI. Cardiology NPs, fellows, and residents were asked to use the risk-assessment form to calculate patients' risk for readmission, and refer patients to the post-Ml clinic if an appointment with a cardiologist was not available within 7 to 14 days after discharge. Every week during the intervention phase, the investigator sent reminder emails to ensure form completion. Providers were asked to calculate and write the score, the discharge and referral dates, where referrals were made (a cardiologist or the post-MI clinic), date of appointment, and reason for not scheduling an appointment or not referring on the risk assessment form, and to drop the completed forms in specific labeled boxes located at the CCU and PCCU work stations. The investigator collected the completed forms weekly. When the number of discharged patients did not match the number of completed forms, the investigator followed up with discharging providers to understand why.

\section{Data and Data Collection}

Data to determine whether the use of the new protocol increased discharge referrals among patients with type I
Ml within the recommended timeframes were collected by electronic chart review. Data included discharging unit, patients' age, gender, admission and discharge date, diagnosis, referral to a cardiologist and the post-MI clinic, and appointment date. Clinical data needed to calculate the AMI READMITS score was also collected: $\mathrm{PCl}$ within 24 hours, serum creatinine, systolic blood pressure (SBP), brain natriuretic peptide (BNP), and diabetes status.

Data to assess provider satisfaction with the usability and usefulness of the new protocol were gathered through an online survey. The survey included 1 question related to the providers' role, 1 question asking whether they used the risk assessment for each patient, and 5 Likert-items assessing the ease of usage. An additional open-ended question asked providers to share feedback related to integrating the AMI READMITS risk assessment score to the post-MI referral protocol long term.

To evaluate how consistently providers utilized the new referral protocol when discharging patients with type I MI, the number of completed forms was compared with the number of those patients who were discharged.

\section{Statistical Analysis}

Descriptive statistics were used to summarize patient demographics and to calculate the frequency of referrals before and during the intervention. Chi-square statistics were calculated to determine whether the change in percentage of referrals and timely referrals was significant. Descriptive statistics were used to determine the level of provider satisfaction related to each survey item. A content analysis method was used to synthesize themes from the open-ended question asking clinicians to share their feedback related to the new protocol.

\section{Results}

Fifty-seven patients met the study inclusion criteria: 29 patients during the preintervention phase and 28 patients during the intervention phase. There were 35 male (61.4\%) and 22 female (38.6\%) patients. Twentyfive patients (43.9\%) were from age groups 41 through 60 years and 61 through 80 years, respectively, repre- 
senting the majority of included patients. Seven patients (12.3\%) were from the 81 years and older age group. There were no patients in the age group 18 through 40 years. Based on the AMI READMITS score calculation, $57.9 \%(n=33)$ patients were from a low-risk group (includes extremely low and low risk for readmission) and $42.1 \%(n=24)$ were from a high-risk group (includes moderate, high, and extremely high risk for readmission).

Provider adoption of the new protocol during the intervention was high. Referral forms were completed for $82 \%$ ( $n=23$ ) of the 28 patients during the intervention. Analysis findings showed a statistically significant increase in documented referrals after implementing the new referral protocol. During the preintervention phase, $66 \%$ ( $n=19$ ) of patients with type I MI were referred to see a cardiologist or an NP at a post-Ml clinic and there was no documented referral for $34 \%(n=10)$ of patients. During the intervention phase, $89 \%(n=25)$ of patients were referred and there was no documented referral for $11 \%(n=3)$ of patients. Chi-square results indicated that the increase in referrals was significant $\chi^{2}=4.571$, $d f=1, P=0.033$.

Data analysis examined whether patient referrals fell within the recommended timeframe of 7 days for the high-risk group (included moderate-to-extremely high risk) and 14 days for the low-risk group (included lowto-extremely low risk). During the preintervention phase, $31 \%(n=9)$ of patient referrals were scheduled as recommended; $28 \%(n=8)$ of patient referrals were scheduled but delayed; and there was no referral date documented for $41 \%(n=12)$ of patients. During the intervention phase, referrals scheduled as recommended increased to $53 \%(n=15) ; 25 \%(n=7)$ of referrals were scheduled but delayed; and there was no referral date documented for $21.4 \%(n=6)$ of patients. The change in appointments scheduled as recommended was not significant $(\chi 2=3.550, d f=2, P=0.169)$.

Surveys were emailed to 25 cardiology fellows and 3 cardiology NPs who participated in this study. Eighteen of the 28 clinicians (15 cardiology fellows and 3 cardiology NPs) responded for a response rate of $64 \%$. One of several residents who rotated through the CCU and PCCU during the intervention also completed the survey, for a total of 19 participants. When asked if the protocol was easy to use, $79 \%$ agreed or strongly agreed. Eighteen of the 19 participants (95\%) agreed or strongly agreed that the protocol was useful in making referral decisions. Sixty-eight percent agreed or strongly agreed that the AMI READMITS risk assessment score improves referral process. All participants agreed or strongly agreed that there should be an option to incorporate the AMI READMITS risk assessment score into electronic clinical notes. When asked whether the AMI READMITS risk score should be implemented in clinical practice, responses were mixed (Figure 3). A common theme among the 4 participants who responded with comments was the need for additional data to validate the usefulness of the AMI READMITS to reduce readmissions. In addition, 1 participant commented that "manual calculation [of the risk score] is not ideal."

\section{Discussion}

This project demonstrated that implementing an evidence-based referral protocol integrating the AMIREADMITS score can increase timely postdischarge referrals among patients with type I MI. The percentage of appropriately scheduled appointments increased during the intervention phase; however, a relatively high number of appointments were scheduled outside of the recommended timeframe, similar to preintervention. Thus, while the new protocol increased referrals and provider documentation of these referrals, it appears that challenges in scheduling timely referral appointments remained. This project did not examine the reasons for delayed appointments.

The survey findings indicated that providers were generally satisfied with the usability and usefulness of the new risk assessment protocol. A large majority agreed or strongly agreed that it was easy to use and useful in making referral decisions, and most agreed or strongly agreed that it improves the referral process. Mixed opinions regarding implementing the AMI READMITS score in clinical practice, combined with qualitative findings, suggest that a lack of external validation of the AMI READMITS presents a barrier to its long-term adoption. All providers who participated in the survey agreed or strongly agreed that the risk 


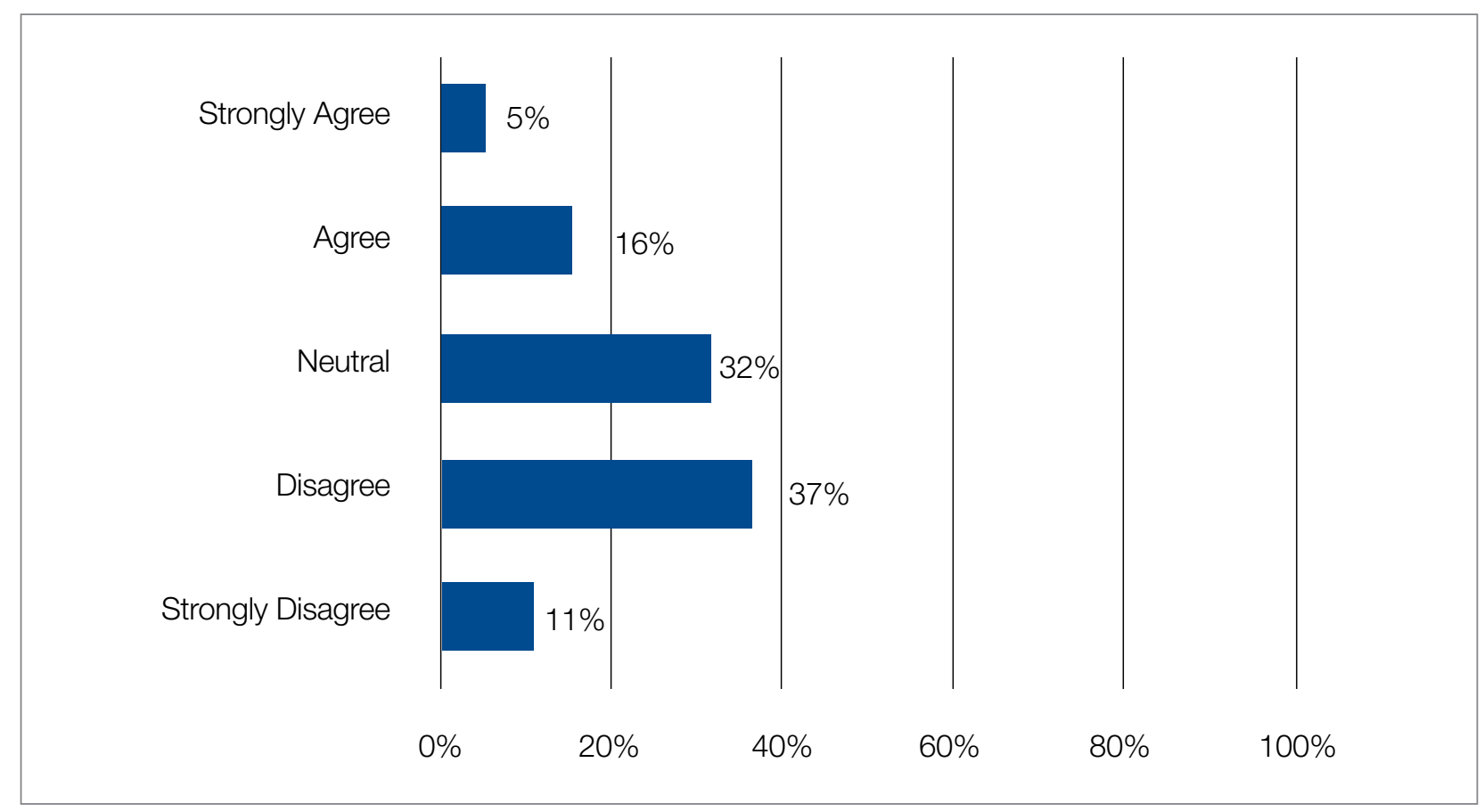

Figure 3. Provider perceptions related to implementing the AMI READMITS score in clinical practice. While overall responses do not show a clear predominance of a specific opinion, more providers disagreed or strongly disagreed, 9 (48\%), than agreed or strongly agreed, 4 (21\%), with implementing the score in clinical practice.

assessment should be incorporated into electronic clinical notes. We have begun the process of working with the EHR vendor to automate the AMl risk-assessment within the referral work-flow, which will provide an opportunity for a follow-up quality improvement study.

This quality improvement project has several limitations. First, it implemented a small change in 2 inpatient units at 1 hospital using a simple pre- posttest design. Therefore, the findings are not generalizable to other settings. Prior to the intervention, some referrals may have been made without documentation. While the authors were able to trace undocumented referrals for patients who were referred to the post-Ml clinic or to a cardiologist affiliated with the hospital, some patients may have been referred to cardiologists who were not affiliated with the hospital. Another limitation was that the self-created provider survey used was not tested in other clinical settings; thus, it cannot be determined whether the sensitivity and specificity of the survey questions are high. In addition, the clinical providers who participated in the study knew the study team, which may have influenced their behavior during the study period. Furthermore, the identified improvement in clinicians' referral practices may not be sustainable due to the complexity and effort required to manually calculate the risk score. This limitation could be eliminated by integrating the risk score calculation into the EHR.

\section{Conclusion}

Early follow-up after discharge plays an important role in supporting patients' self-management of some risk factors (ie, diet, weight, and smoking) and identifying gaps in postdischarge care which may lead to readmission. This project provides evidence that integrating the AMI READMITS risk assessment score into the referral process can help to guide discharge decision-making and increase timely, appropriate referrals for patients with MI. Integration of a specific risk assessment, such as the AMI READMITS, within the post-MI referral protocol may help clinicians make more efficient, educated referral decisions. Future studies should explore more 
specifically how and why the new protocol impacts clinicians' decision-making and behavior related to post-Ml referrals. In addition, future studies should investigate challenges associated with scheduling postdischarge appointments. It will be important to investigate how integration of the new protocol within the EHR may increase efficiency, consistency, and provider satisfaction with the new referral process. Additional research investigating the effects of the AMI READMITS score on readmissions reduction will be important to promote long-term adoption of the improved referral protocol in clinical practice.

Acknowledgments: The authors thank Shelly Conaway, ANP-BC, $M S N$, Angela Street, ANP-BC, MSN, Andrew Geis, ACNP-BC, MSN, Richard P. Jones II, MD, Eunice Young, MD, Joy Rothwell, $M S N, R N-B C$, Allison Olazo, MBA, MSN, RN-BC, Elizabeth Heck, $R N-B C$, and Matthew Trojanowski, MHA, MS, RRT, CSSBB for their support of this study.

Corresponding author: Nailya Muganlinskaya, DNP, MPH, ACNPBC, MSN, The Johns Hopkins Hospital, 1800 Orleans St, Baltimore, MD 21287; nmuganl1@jhmi.edu.

Financial disclosures: None.

\section{References}

1. Why it is important to improve care transitions? Society of Hospital Medicine. Accessed June 15, 2020. https://www.hospitalmedicine. org/clinical-topics/care-transitions/

2. Tong L, Arnold T, Yang J, et al. The association between outpatient follow-up visits and all-cause non-elective 30-day read- missions: a retrospective observational cohort study. PloS One. 2018;13(7):e0200691.

3. Jackson C, Shahsahebi M, Wedlake T, DuBard CA. Timeliness of outpatient follow-up: an evidence-based approach for planning after hospital discharge. Ann Fam Med. 2015;13(2):115-22.

4. Health Research \& Educational Trust. Preventable Readmissions Change Package. American Hospital Association. Updated December 2015. Accessed June 10, 2020. https://www. aha.org/sites/default/files/hiin/HRETHEN_ChangePackage_ Readmissions.pdf

5. Tung Y-C, Chang G-M, Chang $\mathrm{H}-\mathrm{Y}$, Yu T-H. Relationship between early physician follow-up and 30-day readmission after acute myocardial infarction and heart failure. Plos One. 2017;12(1):e0170061.

6. Kaplan RM, Koehler J, Zieger PD, et al. Stroke risk as a function of atrial fibrillation duration and $\mathrm{CHA}_{2} \mathrm{DS}_{2}$-VASc score. Circulation. 2019;140(20):1639-46.

7. Balan P, Zhao Y, Johnson S, et al. The Society of Thoracic Surgery Risk Score as a predictor of 30-day mortality in transcatheter vs surgical aortic valve replacement: a single-center experience and its implications for the development of a TAVR risk-prediction model. J Invasive Cardiol. 2017;29(3):109-14.

8. Smith LN, Makam AN, Darden D, et al. Acute myocardial infarction readmission risk prediction models: A systematic review of model performance. Circ Cardiovasc Qual Outcomes. 2018; 11(1):e003885.

9. Baker H, Oliver-McNeil S, Deng L, Hummel SL. See you in 7: regional hospital collaboration and outcomes in Medicare heart failure patients. JACC Heart Fail. 2015;3(10):765-73.

10. Batten A, Jaeger C, Griffen D, et al. See you in 7: improving acute myocardial infarction follow-up care. BMJ Open Qual. 2018;7(2):e000296.

11. Lee DW, Armistead L, Coleman H, et al. Abstract 15387: Postdischarge follow-up within 14 days reduces 30-day hospital readmission rates in patients with acute myocardial infarction and/or acutely decompensated heart failure. Circulation. 2018;134 (1):A 15387.

12. Thygesen K, Alpert JS, Jaffe AS, et al. Fourth universal definition of myocardial infarction. Circulation. 2018;138 (20):e:618-51. 